\title{
Universiteit
}

Leiden

The Netherlands

\section{Determinants of absence of osteoarthritis in old age}

Goekoop, R.J.; Kloppenburg, M.; Kroon, H.M.; Dirkse, L.E.V.; Huizinga, T.W.J.; Westendorp, R.G.J.; Gussekloo, J.

\section{Citation}

Goekoop, R. J., Kloppenburg, M., Kroon, H. M., Dirkse, L. E. V., Huizinga, T. W. J., Westendorp, R. G. J., \& Gussekloo, J. (2011). Determinants of absence of osteoarthritis in old age. Scandinavian Journal Of Rheumatology, 40(1), 68-73.

doi:10.3109/03009742.2010.500618

Version: $\quad$ Not Applicable (or Unknown)

License: $\quad$ Leiden University Non-exclusive license

Downloaded from: https://hdl.handle.net/1887/117606

Note: To cite this publication please use the final published version (if applicable). 


\title{
Determinants of absence of osteoarthritis in old age
}

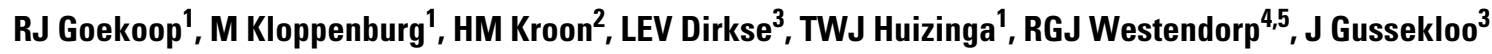 \\ Departments of ${ }^{1}$ Rheumatology, ${ }^{2}$ Radiology, ${ }^{3}$ Public Health and Primary Care, ${ }^{4}$ Gerontology and Geriatrics, Leiden University Medical \\ Centre, Leiden, ${ }^{5}$ Netherlands Consortium of Healthy Ageing, Leiden, The Netherlands
}

\begin{abstract}
Objective: To investigate factors associated with absence of osteoarthritis (OA).
Methods: In 82 well-functioning 90-year-old participants from a cross-sectional birth cohort, radiographs of hands, hips, and knees were acquired and scored according to the Kellgren and Lawrence (K-L) method for determining OA. A score of $\geq 2$ was considered as OA. 'Free from OA' was defined as no hip or knee OA and less than three hand joints with OA. Logistic regression analyses were used to investigate associations with absence of OA.

Results: Absence of hip, knee, and hand OA was seen in 63, 51, and 29\% of participants, respectively. Joints on the left and right side of the body were equally affected. Sixteen per cent of 90 -year old participants were 'free from OA'. Absence of knee OA was associated with being male. A family history of finger nodes was negatively associated with absence of hip and hand OA. Body mass index (BMI) was negatively associated with 'free from OA', and also with absence of hip and knee OA. A history of heavy occupational work was associated with 'free from OA' [odds ratio (OR) 7.2, 95\% confidence interval (CI) 1.3-39.9] and with absence of hand OA in particular (OR 2.7, 95\% CI 1.0-7.1). Conclusions: In 90-year-olds, absence of OA is associated with male sex, a normal BMI, absence of familial predisposition for OA, and, contrary to our expectation, heavy work. Further research in protective genetic factors is needed.
\end{abstract}

Osteoarthritis $(\mathrm{OA})$ is a chronic joint disorder characterized by articular cartilage destruction and changes in subchondral bone that can occur in any joint, but has a preference for hands, hips, and knees and is often polyarticular. It is a major cause of disability worldwide. Although the exact pathogenesis of OA remains unknown, OA is regarded as a disorder that is the result of a complex interplay of a systemic predisposition, including genetic, hormonal, and metabolic factors, and local biomechanical factors (1).

The prevalence of OA increases with age, which is the most prominent risk factor for the condition. The prevalence of OA in subjects $\geq 90$ years old is unknown. The prevalence of radiographic OA in men and women over 80 years of age is the range $26-55 \%$ for knees, $11-26 \%$ for hips, and 48-73\% for distal interphalangeal joints (DIPJs) $(2,3)$. Indeed, OA is often regarded as an inevitable, degenerative disease of ageing. However, the prevalence of OA reaches a plateau, and even decreases after the seventh decade of life, as is shown in population-based studies where a range of age groups were included $(2,4)$. The incidence of OA also declines around the age of 80 . The cause of the decline in prevalence is unknown.

One explanation for the levelling off of OA development in old age is the presence of protective factors, that

\footnotetext{
Robbert J Goekoop, Department of Rheumatology, Leiden University Medical Centre, Leiden, The Netherlands.

E-mail: r.j.goekoop@lumc.nl
}

Accepted 9 June 2010 is factors that might coincide with longevity (5). Hence, it is of particular interest to investigate whether factors can be identified that are associated with absence of OA, especially at multiple joint sites. Because little is known about true protective factors against OA, we speculate that the absence of known risk factors can serve as protective factors. Factors expected to be associated with absence of OA are male sex, normal weight, occupational work without heavy loading, absence of history of meniscectomy or trauma, smoking, and absence of a familial predisposition (1).

To prevent misclassification, absence of OA can best be studied at very old age. Comparative data about prevalence rates of absence of OA at multiple joint sites are lacking at this age. Therefore, in a cross-sectional study design, we examined the OA status of the hands, hips, and knees of individuals $\geq 90$ years of age, and we investigated which factors were associated with the absence of OA, which could confer protection. Insight into possible protective factors for OA will increase the understanding of the pathogenesis of OA in general.

\section{Materials and methods}

\section{Study population}

The Leiden 85-plus Study is a prospective populationbased study of all 85-year-old inhabitants of Leiden, The Netherlands. The study design and characteristics of the 
cohort have been described in detail elsewhere (6). In short, between September 1997 and September 1999 all 705 members of the 1912-1914 birth cohorts in the city of Leiden were invited to participate in a study of the health of the very elderly during the month after their 85 th birthday. The initial study cohort consisted of 599 participants ( $87 \%$ response rate) at age 85 years. There were no selection criteria related to health or demographic characteristics. In April 2004 all participants of the Leiden 85plus Study (age range 89-91 years, 90 years on average) were invited to take part in the present subsidiary study. Participants gave informed consent to enter the study but for people who were severely cognitively impaired, a guardian gave informed consent. The Medical Ethics Commission of Leiden University approved the study.

\section{Demographic and clinical characteristics}

Demographic data and medical history were collected when participants were aged 85 years. When participants reached age 90, a research nurse visited them at home and collected information by questionnaire on demographic characteristics and risk factors for OA, including gender, obesity, joint injury, a history of heavy occupational work, smoking, and congenital or developmental disorders. Their medical history, regarding OA ('history of OA'), rheumatoid arthritis (RA) ('history of RA') and co-morbid conditions, was obtained from participants' general practitioners (GPs) or nursing-home physicians by standardized interviews. In participants with radiographic examination, a history of heavy occupational work was assessed by a standardized questionnaire of established occupational risk factors of OA: intensive standing or walking, lifting heavy objects, dealing with heavy machinery, and bending and kneeling during their main occupation (7). If one or more conditions were present during their main occupation, they were designated as 'heavy occupational work'. A familial history of OA was assessed by asking whether their parents had 'nodes on their fingers'. Cognitive function, included in the analysis to give insight into possible reasons for not attending the study centre, was assessed in all participants with the Mini-Mental State Examination (MMSE) (8). Disability in basic activities of daily living (ADL) was measured using the Groningen Activity Restriction Scale (GARS; range 18-72; a low score reflects high functional ability) (9). A low level of education was defined as primary school level, or less. Their height and weight were measured by the research nurse.

\section{Radiographic examination and scoring}

Radiographs of hands (dorso-volar), hips (anteriorposterior, supine), and knees (weight-bearing, posterioranterior, full-extension) were obtained according to a standardized method with a fixed film-focus distance and a fixed position of the joint.
Radiographs were assessed for OA by two independent readers (HMK and RJG), who were blinded to patient characteristics. The method of scoring OA followed that described by Kellgren and Lawrence (K-L) (score range 0-4) (10). The following joints were scored: eight DIPJs, eight proximal IPJs (PIPJs), two first IPJs, and two first carpometacarpal joints (CMCJs), both hips, and both femoro-tibial joints.

The interobserver reliability for a K-L score $\geq 2$ as a dichotomous variable expressed by the $\kappa$ statistic varied from 0.88 for the right hip to 0.41 for the right first IPJ. In cases of disagreement between readers' evaluation of the radiographic OA status of a joint, a rescore was performed to reach consensus, which was used for calculations.

\section{OA diagnosis}

$\mathrm{OA}$ for each joint was defined as a $\mathrm{K}-\mathrm{L}$ score $\geq 2$. The presence of hand OA was defined as a K-L score of $\geq 2$ in three or more joints of all the scored hand joints. This cut-off was chosen because radiological OA in one or two hand joints could be the result of single traumatic lesions. This study focused on the systemic pathophysiology of OA, hence single lesions potentially due to trauma alone were considered outside the scope of this paper. A participant was considered 'free from $\mathrm{OA}^{\prime}$ when no OA was present in hips or knees, and OA in less than three hand joints. A joint prosthesis in a particular joint was regarded as $\mathrm{OA}$ if this was the indication for the operation. If the reason was unknown, the joint was not included in the rating.

\section{Statistical analyses}

Differences in demographic and clinical characteristics between participants who were able to visit the study centre for radiographic examination versus those who were not, and between participants with OA versus those without OA were determined using two-sided independent T-tests for continuous variables and $\chi^{2}$ tests for non-continuous variables. To investigate the association between risk factors and OA, logistic regression analysis was performed and odds ratios (ORs) with $95 \%$ confidence intervals (CIs) were calculated. Adjustments were made for gender and tertiles of BMI, a history of heavy occupational work, current or previous smoking, and finger nodes in the family.

All analyses were carried out using SPSS version 16.0 (SPSS Inc, Chicago, IL, USA).

\section{Results}

\section{Study population}

Two hundred and ninety-one elderly individuals were eligible to take part in the present study. Of those, 258 
(A)

Flowchart at 85-89 years

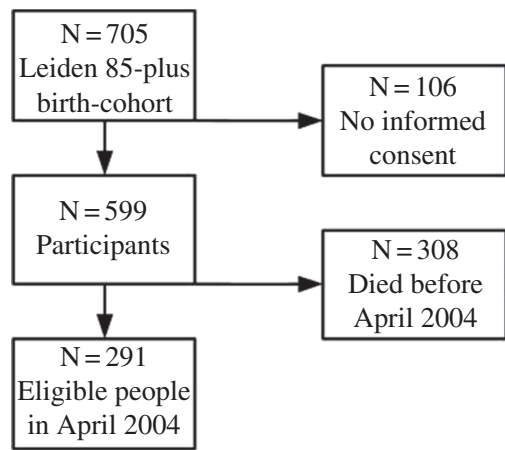

(B)

Flowchart at 90 years

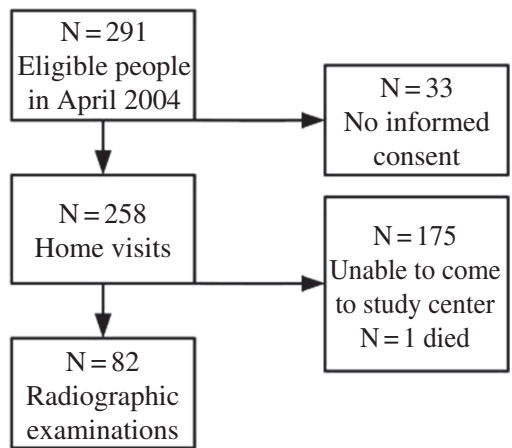

Figure 1. Flowchart at (A) 85-89 years and (B) 90 years.
(89\%) were willing to participate and were visited in their home to collect data. Eighty-two (32\%) participants were able to visit the study centre for radiographic examinations (Figure 1).

Table 1 shows the demographic and clinical characteristics of the participants, divided into those who were able to attend for radiographic examination $(n=82)$ and those who were not $(n=176)$. Table 1B presents the same characteristics, dividing participants into those with versus those without OA.

The majority of study participants were women and, overall, participants were slightly overweight. Nearly one-third of all participants had a 'history of OA', equally distributed among those with versus those without radiographic examination. However, between-group differences were observed; that is those who did not undergo radiographic examination had lower cognitive function (MMSE), took part in fewer daily life activities (GARS), and were less educated. Dementia was the only comorbidity to be less prevalent in those with than in those without radiographic examination $(1.2 \%$ vs. $8.8 \%)$.

\section{Distribution and absence of $\mathrm{OA}$}

Figure 2 describes the point prevalences of radiographically determined OA in the joints of men and women, according to the right and left sides of the body. No difference was detected in the prevalence of OA in joints on the right and left side of the body for either men or women. DIPJs of the second digit, first IPJs, and first CMCJs were affected most frequently by OA. Women had a higher, although not statistically significant, total number of OA joints (median 7, range 0-22) compared to men (median 4, range 0-19).

Hip prostheses were implanted in $11(13.4 \%)$ participants on the right side of their body: due to OA in seven participants, following hip fractures in two, and for unknown reasons in two participants. Three (3.7\%) participants had hip prostheses on the left side of their body: two because of OA, and one for an unknown reason. Knee prostheses were present on the right side of the body in four (4.9\%) participants, because of OA in three and for an unknown reason in one participant, and

Table 1. Sociodemographics, clinical characteristics, and functional status of participants, categorized according to (A) those who underwent radiographic examination and those who did not, and (B) those with versus those without osteoarthritis (OA).

\begin{tabular}{|c|c|c|c|c|c|c|}
\hline \multirow[b]{2}{*}{ Characteristics } & \multicolumn{3}{|c|}{ (A) Radiographic examination } & \multicolumn{3}{|c|}{ (B) Osteoarthritis } \\
\hline & Yes ( $n=82)$ & No $(n=176)$ & $p$-value & Yes $(n=69)$ & No $(n=13)$ & $\mathrm{p}$-value \\
\hline \multicolumn{7}{|l|}{ Sociodemographic characteristics } \\
\hline Age (years), mean \pm SD & $90.4 \pm 0.6$ & $90.6 \pm 0.5$ & 0.98 & & & \\
\hline Male gender, $\mathrm{n}(\%)$ & $27(32.9)$ & $41(23.4)$ & 0.13 & $20(29)$ & $7(54)$ & 0.08 \\
\hline Low level of education, $\mathrm{n}(\%)$ & $39(47.6)$ & $120(68.2)$ & 0.002 & $33(48)$ & $6(46)$ & 0.58 \\
\hline Current or previous smoking, $\mathrm{n}(\%)$ & $37(45.1)$ & $73(41.7)$ & 0.72 & $29(42)$ & $8(62)$ & 0.15 \\
\hline \multicolumn{7}{|l|}{ Clinical characteristics } \\
\hline History of $0 A, n(\%)$ & $24(29.3)$ & $56(31.8)$ & 0.68 & $24(35)$ & $0(0)$ & $<0.01$ \\
\hline History of hip fracture, $\mathrm{n}(\%)$ & $9(11.0)$ & $17(9.7)$ & 0.74 & $7(10)$ & $2(15)$ & 0.44 \\
\hline History of heavy occupational work, $\mathrm{n}(\%)$ & $34(41.5)$ & NA & - & $25(36)$ & $9(69)$ & 0.03 \\
\hline BMI $\left(\mathrm{kg} / \mathrm{m}^{2}\right)$, mean \pm SD & $26.8 \pm 4.3^{*}$ & $27.1 \pm 4.7 \dagger$ & 0.64 & $27.2 \pm 4.38 \ddagger$ & $24.8 \pm 2.88$ & 0.06 \\
\hline Family history of finger nodes§, $\mathrm{n}(\%)$ & - & - & - & $12(20)$ & $1(8)$ & 0.07 \\
\hline \multicolumn{7}{|l|}{ Functional status } \\
\hline MMSE, median (IOR) & $28(26-29)$ & $22(16-27)$ & $<0.001$ & $28.0(26.0-29.0)$ & $28.0(26.0-28.5)$ & 0.91 \\
\hline GARS, median (IOR) & $33(25-42)$ & $48(34-60)$ & $<0.001$ & $34.0(27.5-41)$ & $25.0(21-35)$ & 0.03 \\
\hline
\end{tabular}

MMSE, Mini-Mental State Examination; GARS, Groningen Activity Restriction Scale; BMI, body mass index; IQR, interquartile range; $N A$, not available; SD, standard deviation.

${ }^{*} \mathrm{n}=78 ; \mathrm{tn}=144 ; \ddagger \mathrm{n}=65$. §BMI data available for 78 of 82 participants. 
Point Prevalence of OA in 55 90-year-old women, stratified for joint side.

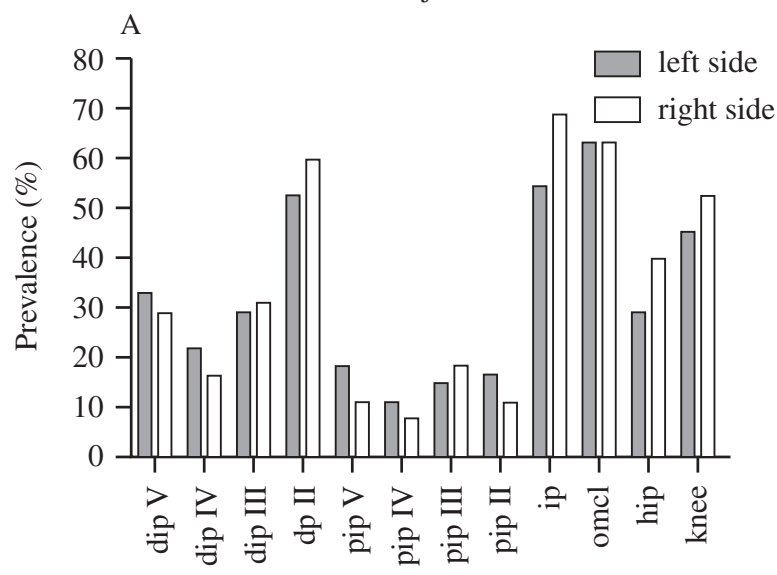

Joint
Point Prevalence of OA in 27 90-year-old men, stratified for joint side.

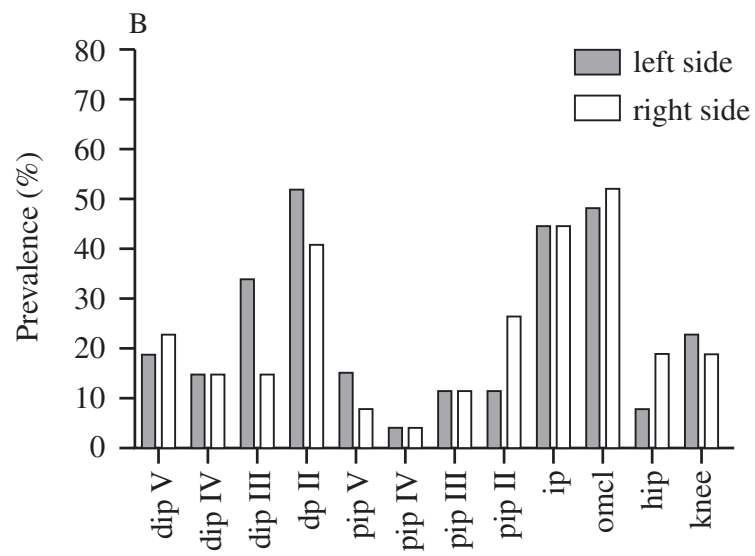

Joint

Figure 2. Point prevalence of radiographic osteoarthritis in (A) 55 female and (B) 27 male 90-year-olds stratified for joint side.

in two (2.4\%) participants on the left side of the body, both because of OA.

Only two participants had a history of unilateral menisectomy: OA was present in one, the other participant had knee prosthesis. The majority of participants had no hip $(63.4 \%)$ or knee OA $(51.2 \%)$. Hand OA was absent in $29.3 \%$ of participants. Thirteen $(15.9 \%)$ participants were 'free from OA'. Absence of any form of OA was found in eight $(9.8 \%)$ participants.

Factors associated with the absence of $0 \mathrm{~A}$ in individual joint groups

The demographic and clinical characteristics associated with the absence $\mathrm{OA}$ in the hands, hips, and knees are depicted in Table 2. Being male predisposed subjects to the absence of knee OA (adjusted OR 8.4, 95\% CI 1.9-38.4) but not to the absence of hip OA (adjusted OR $1.5,95 \%$ CI $0.28-8.2$ ) or to the absence hand OA (adjusted OR 0.93, 95\% CI 0.25-3.4). A family history of finger nodes reduced the change to be free from hip OA (OR 0.12, 95\% CI 0.02-0.66); the same trend was observed for hand OA.

In a dose-dependent manner, a higher BMI was associated with a lower chance of absence of knee OA (OR for the highest tertile of BMI versus the lowest tertile for absence of knee OA 0.23 , 95\% CI 0.06-0.95) and absence of hip OA (OR for the highest tertile of BMI versus the lowest tertile for absence of hip OA $0.03,95 \%$ CI 0.01-0.30). Borderline statistical significance was found for the negative relationship between the highest tertile of BMI and absence of hand OA. The association between heavy occupational work and the absence of OA in individual joint groups was seen for the hand (adjusted OR 3.3, 95\% CI 0.96-11.1) but not for the knee or hip.

\section{Factors associated with 'free from $0 A^{\prime}$}

The associations between participants' demographic and clinical characteristics and being 'free from $\mathrm{OA}^{\prime}$ ' are shown in Table 2. None of the participants who were 'free from $\mathrm{OA}^{\prime}$ ' had a family history of finger nodes. Because of the low numbers statistical significance was not reached. BMI was associated with the absence of $\mathrm{OA}$ in a dose-dependent manner (OR for absence of OA in the highest tertile of BMI compared to the lowest tertile $0.06,95 \%$ CI $0.007-0.51$ ). Heavy occupational work was associated with the absence of OA (OR 7.2, 95\% CI 1.3-39.9). Level of education, a possible confounder, did not differ between participants with and without OA (OR $1.06,95 \%$ CI $0.33-3.4)$. When all associations were investigated in 90-year-olds without any form of OA $(n=8)$ versus all others $(\mathrm{n}=74)$, similar the results were obtained (supplementary tables are available on request).

\section{Discussion}

Factors associated with the absence of OA were investigated in a convenience sample of 90 -year-olds. Of the well-functioning participants in the present study, $10 \%$ had no OA at all and $16 \%$ were only slightly affected (two hand joints or fewer) with OA. The majority of participants had neither knee nor hip OA. In the current study hereditary factors seem to play a major role in the preservation of healthy joints: absence of hip OA and, to a lesser extent, absence of hand OA and 'free from OA' were associated with the absence of a family history of hand OA. In accordance with findings in younger age groups, a BMI $<24.8 \mathrm{~kg} / \mathrm{m}^{2}$ and male gender were associated with the absence of knee OA; however, in contrast to findings in younger age groups, a strong association was also seen between a low BMI 
Table 2. Associations for absence of (joint group) OA.

\begin{tabular}{|c|c|c|}
\hline Characteristic & $\begin{array}{l}\text { Adjusted OR } \\
(95 \% \mathrm{Cl})^{*}\end{array}$ & p-valu \\
\hline \multicolumn{3}{|l|}{ Absence of knee $0 \mathrm{~A}$} \\
\hline Male gender & $8.4(1.9-38.4)$ & 0.006 \\
\hline $\begin{array}{l}\text { Family history of finger nodes } \dagger \\
\left.\text { BMI (ref. } 18.9-24.8 \mathrm{~kg} / \mathrm{m}^{2}\right) \ddagger\end{array}$ & $0.80(0.19-3.3)$ & 0.76 \\
\hline $24.9-27.9 \mathrm{~kg} / \mathrm{m}^{2}$ & $0.18(0.04-0.74)$ & 0.02 \\
\hline $28.0-41.9 \mathrm{~kg} / \mathrm{m}^{2}$ & $0.23(0.06-0.95)$ & 0.04 \\
\hline History of heavy physical work & $0.46(0.14-1.5)$ & 0.21 \\
\hline Current or previous smoking & $0.32(0.07-1.4)$ & 0.14 \\
\hline \multicolumn{3}{|l|}{ Absence of hip 0A } \\
\hline Male gender & $1.5(0.28-8.2)$ & 0.62 \\
\hline $\begin{array}{l}\text { Family history of finger nodes } † \\
\left.\text { BMI (ref. } 18.9-24.8 \mathrm{~kg} / \mathrm{m}^{2}\right) \ddagger\end{array}$ & $0.12(0.02-0.66)$ & 0.02 \\
\hline $24.9-27.9 \mathrm{~kg} / \mathrm{m}^{2}$ & $0.20(0.03-1.3)$ & 0.10 \\
\hline $28.0-41.9 \mathrm{~kg} / \mathrm{m}^{2}$ & $0.03(0.01-0.30)$ & 0.003 \\
\hline History of heavy physic & $0.66(0.17-2.7)$ & 0.57 \\
\hline Current or previous smoking & $6.2(0.88-43.6)$ & 0.07 \\
\hline \multicolumn{3}{|l|}{ Absence of hand $O A$} \\
\hline Male gender & $0.93(0.25-3.4)$ & 0.93 \\
\hline $\begin{array}{l}\text { Family history of finger nodes } \dagger \\
\left.\text { BMI (ref. } 18.9-24.8 \mathrm{~kg} / \mathrm{m}^{2}\right) \ddagger\end{array}$ & $0.12(0.01-1.1)$ & 0.06 \\
\hline $24.9-27.9 \mathrm{~kg} / \mathrm{m}^{2}$ & $0.73(0.18-2.9)$ & 0.66 \\
\hline $28.0-41.9 \mathrm{~kg} / \mathrm{m}^{2}$ & $0.29(0.07-1.3)$ & 0.10 \\
\hline History of heavy physic & $3.3(0.96-11.1)$ & 0.06 \\
\hline Current or previous smoking & $1.4(0.36-5.2)$ & 0.65 \\
\hline \multicolumn{3}{|l|}{ ‘Free from $0 A^{\prime}$} \\
\hline Male gender & $3.0(0.58-16.0)$ & 0.19 \\
\hline $\begin{array}{l}\text { Family history of finger nodes } \dagger \\
\left.\text { BMI (ref. } 18.9-24.8 \mathrm{~kg} / \mathrm{m}^{2}\right) \ddagger\end{array}$ & $0.22(0.02-2.1)$ & 0.19 \\
\hline $24.9-27.9 \mathrm{~kg} / \mathrm{m}^{2}$ & $0.24(0.04-1.5)$ & 0.12 \\
\hline $28.0-41.9 \mathrm{~kg} / \mathrm{m}^{2}$ & $0.06(0.007-0.51)$ & 0.01 \\
\hline History of heavy physical work & $7.2(1.3-39.9)$ & 0.03 \\
\hline Current or previous smoking & $0.72(0.13-4.0)$ & 0.71 \\
\hline
\end{tabular}

$\mathrm{OR}, \mathrm{Odds}$ ratio; $\mathrm{Cl}$, confidence interval; $\mathrm{BMI}$, body mass index. $O R$ sepresent the relative risk of participants without joint group $0 \mathrm{~A}$ versus those with joint group $0 \mathrm{~A}$ (reference group) with respect to the respective characteristic.

${ }^{*}$ Adjustments to ORs were made for gender, tertiles of BMI, a history of heavy occupational work, current or previous smoking, and family history of finger nodes.

tFamily history of finger nodes: data available in 73 of 82 participants.

$\ddagger B M I$ available in 78 of 82 participants.

and the absence of hip OA (11-13). Furthermore, two observations suggest that $\mathrm{OA}$ found in the general population in very old age is not an inevitable consequence of ageing and weight loading: first, no difference in OA between the left and right side of the body was observed, and second, heavy occupational work was associated with the absence rather than the presence of OA.

This unique cohort consisted of 90-year-old individuals. In this cohort extensive radiographic assessment involved multiple joint sites, leading to an investigation of the systemic condition of absence of OA at the level of the patient, a situation that we called 'free from OA'. Hence, the present study provides an opportunity to investigate the preservation of healthy joints and the protection of joints against OA, which can increase our understanding of the pathophysiology of OA.

The present study unexpectedly showed that a history of heavy occupational work was positively associated with the absence of OA, and hence the preservation of healthy joints. Furthermore, analyses of OA in individual joint groups demonstrated that a history of heavy occupational work was associated with the absence of hand OA. This somewhat surprising finding, contrary to past literature (7), might be explained by the probable presence of protective factors. Even with a history of heavy occupational work, which probably caused microtrauma to the joint throughout life, no OA developed as a consequence. It may be that, in our population of 90year-olds, genetic factors encoding for tissue repair mechanisms were present that preserved healthy joints. We could speculate that these factors also influenced the individuals' longer than average survival (5).

We have shown that a BMI below $24.8 \mathrm{~kg} / \mathrm{m}^{2}$ is associated with the absence of OA. It is also noteworthy that not only was a low BMI associated with the absence of knee OA, as in younger age groups (14), but also a strong association was demonstrated for the absence of hip OA. In a systematic review including studies of all age groups, but where the oldest old were under-represented, a moderate association $(\mathrm{OR} \approx 2)$ was reported between being overweight and clinical diagnosis of hip OA (13, 14). However, radiographic hip OA demonstrated no association with BMI. That a clear association between low BMI and the absence of radiographic OA is seen in these oldest old may be explained by many years of being overweight. Although we do not know the BMI of the participants during their entire lifetime, we could speculate that the long-term effect of overweight on hip joints may result in the considerable association between overweight and hip OA seen in the present study. A trend towards an association between BMI and absence of hand OA was also seen. This in accordance with that seen at younger ages (15). Furthermore, the present findings suggest that, in the oldest old, in addition to the mechanical loading effect on joints that being overweight incurs, other factors (metabolic or hormonal) are involved that might be produced by the adipose tissue.

This study has some potential limitations that should be addressed. First, participants who underwent radiographic examinations were a 'convenience sample' of our wider study population. However, the use of a 'convenience sample' did not influence the (GPderived) prevalence of 'history of OA' between those with versus those without radiographic examination. Nevertheless, we accept that participants with a radiographic examination and without OA may be overrepresented. However, this possible over-representation or healthy person effect does not harm the purpose of our explorative study, namely to determine protective factors against OA rather than risk factors in participants with OA. In fact, an over-representation of absence of OA would reduce the effect size of the association. 
Second, the number of participants who underwent radiographic examination was small. However, despite the small sample size, established risk factors, as well as protective factors, for OA were statistically significant, but with broad confidence intervals due to low numbers.

In conclusion, absence of OA, although seldom, was observed in the 90-year-olds participating in this study. This absence was associated with male gender, a normal BMI, a history of heavy occupational work, and absence of a familial predisposition. There was no associated risk with dexterity. These factors show the complex interaction between $\mathrm{OA}$ risk and protective factors, which may encompass genotypic and/or phenotypic elements as well as environmental exposure. This is subject to further investigation.

\section{Acknowledgements}

The Leiden 85-plus Study is a collaborative project of the Department of Gerontology and Geriatrics (RGJ Westendorp) and the Department of Public Health and Primary Care (J Gussekloo) of the Leiden University Medical Centre, Leiden, The Netherlands. RGJ Westendorp is supported by NOI/NOW grants 050-60-810 and 911-03-016.

\section{References}

1. Sharma L, Kapoor D, Issa S. Epidemiology of osteoarthritis: an update. Curr Opin Rheumatol 2006;18:147-56.

2. van Saase JL, van Romunde LK, Cats A, Vandenbroucke JP, Valkenburg HA. Epidemiology of osteoarthritis: Zoetermeer survey. Comparison of radiological osteoarthritis in a Dutch population with that in 10 other populations. Ann Rheum Dis 1989;48:271-80.

3. Bagge E, Bjelle A, Eden S, Svanborg A. Osteoarthritis in the elderly: clinical and radiological findings in 79 and 85 year olds. Ann Rheum Dis 1991;50:535-9.
4. Dahaghin S, Bierma-Zeinstra SM, Ginai AZ, Pols HA, Hazes JM, Koes BW. Prevalence and pattern of radiographic hand osteoarthritis and association with pain and disability (the Rotterdam study). Ann Rheum Dis 2005;64:682-7.

5. Slagboom PE, Heijmans BT, Beekman M, Westendorp RG, Meulenbelt I. Genetics of human aging. The search for genes contributing to human longevity and diseases of the old. Ann N Y Acad Sci 2000;908:50-63.

6. der Wiel AB, van Exel E, de Craen AJ, Gussekloo J, Lagaay AM, Knook DL, et al. A high response is not essential to prevent selection bias: results from the Leiden 85-plus study. J Clin Epidemiol 2002;55:1119-25.

7. Schouten JS, de Bie RA, Swaen G. An update on the relationship between occupational factors and osteoarthritis of the hip and knee. Curr Opin Rheumatol 2002;14:89-92.

8. Tombaugh TN, McIntyre NJ. The mini-mental state examination: a comprehensive review. J Am Geriatr Soc 1992;40:922-35.

9. Kempen GI, Miedema I, Ormel J, Molenaar W. The assessment of disability with the Groningen Activity Restriction Scale. Conceptual framework and psychometric properties. Soc Sci Med 1996;43:1601-10.

10. Kellgren JH. The epidemiology of chronic rheumatism. Volume II: Atlas of standard radiographs of arthritis. Oxford: Blackwell Scientific Publications.

11. Dahaghin S, Bierma-Zeinstra SM, Koes BW, Hazes JM, Pols HA. Do metabolic factors add to the effect of overweight on hand osteoarthritis? The Rotterdam Study. Ann Rheum Dis 2007;66:916-20.

12. Felson DT. Does excess weight cause osteoarthritis and, if so, why? Ann Rheum Dis 1996;55:668-70.

13. Lievense AM, Bierma-Zeinstra SM, Verhagen AP, van Baar ME, Verhaar JA, Koes BW. Influence of obesity on the development of osteoarthritis of the hip: a systematic review. Rheumatology (Oxford) 2002;41:1155-62.

14. Oliveria SA, Felson DT, Cirillo PA, Reed JI, Walker AM. Body weight, body mass index, and incident symptomatic osteoarthritis of the hand, hip, and knee. Epidemiology 1999;10:161-6.

15. Yusuf E, Nelissen R, Ioan-Facsinay A, Stojanovic-Susulic V, Degroot J, van Osch G, et al. Association between weight or body mass index and hand osteoarthritis: a systematic review. Ann Rheum Dis 2010;69:761-5. 